\title{
FISH ACOUSTICS: PHYSICS-BASED MODELING AND MEASUREMENT
}

Davis Benjamin Reeder

Office of Naval Research, Code 32, Arlington, VA, USA., reederd@onr.navy.mil

Follow this and additional works at: https://jmstt.ntou.edu.tw/journal

Part of the Aquaculture and Fisheries Commons

\section{Recommended Citation}

Reeder, Davis Benjamin (2011) "FISH ACOUSTICS: PHYSICS-BASED MODELING AND MEASUREMENT," Journal of Marine Science and Technology. Vol. 19: Iss. 3, Article 6.

DOI: $10.51400 / 2709-6998.2192$

Available at: https://jmstt.ntou.edu.tw/journal/vol19/iss3/6

This Research Article is brought to you for free and open access by Journal of Marine Science and Technology. It has been accepted for inclusion in Journal of Marine Science and Technology by an authorized editor of Journal of Marine Science and Technology. 


\title{
FISH ACOUSTICS: PHYSICS-BASED MODELING AND MEASUREMENT
}

\author{
Davis Benjamin Reeder*
}

Key words: underwater acoustics, fish acoustics, backscatter, target strength, attenuation, measurement, modeling.

\begin{abstract}
The U.S. Office of Naval Research has sponsored research in the area of marine organism acoustics for many years. The research program has included development of theoretical physics-based acoustic scattering models of single animals, high-resolution laboratory measurements of scattering by individual animals, and at-sea field experiments. The program has been focused on the backscattered signal, but has also included investigation of the forward-scattered signal. Downward-looking acoustic surveys using ship borne echosounders rely on the backscattered signal and provide non-invasive, non-destructive, rapid, high-resolution, large area survey capability compared to traditional net tows. Horizontally-oriented acoustic surveys provide the opportunity to investigate both the backscattered and forward-scattered signals from marine organisms and their impact on long-range acoustic propagation characteristics in the shallow water environment. Both cases require an understanding of the scattering characteristics of each type of organism and aggregation in the acoustic path as a function of acoustic frequency and orientation relative to the acoustic source and receiver. This overview of the fish-related acoustics research program includes representative examples which demonstrate the fundamental physical principles which have shaped the program.
\end{abstract}

\section{INTRODUCTION}

A primary motivation for investigation of acoustic scattering by marine organisms is fisheries management. The common objective is knowledge of the distribution, diversity and numerical density of organisms, as well as the ocean environment, habitat and physical processes. Acoustic surveys provide non-invasive, non-destructive, rapid, high-resolution, large area survey capability compared to traditional net tows; however, acoustic surveys provide acoustic data, not biologi-

Paper submitted 05/31/10; revised 07/01/10; accepted 07/16/10. Author for correspondence: Davis Benjamin Reeder (e-mail: reederd@onr.navy.mil). *Office of Naval Research, Code 32, Arlington, VA, USA. cal data. The acoustic backscattered signal must be interpreted for meaningful biological information, such as species identification and size class. This interpretation is difficult because the acoustic signal information is ambiguous, and the biological diversity in the ocean is overwhelming. The strategy is to exploit the amplitude, angle and frequency dependence of the scattered signal to infer the size, shape and material properties of individual scatterers. This assumes each class of organism has a unique "acoustic signature" whose scattering mechanisms can be identified, measured and modeled.

Theoretically, we seek the solution to the acoustic wave equation which governs the propagation of an acoustic wave through a medium with mass.

$$
\nabla^{2} P=\frac{1}{c^{2}} \frac{\partial P}{\partial t^{2}}
$$

The acoustic wave equation relates the divergence of the spatial gradient of the pressure field $(P)$ to the time-evolving pressure field through the sound speed $(c)$ of the medium. The acoustic pressure field is the deviation from the ambient pressure. Specifically, the far-field scattered pressure $\left(P_{\text {scat }}\right)$ is expressed in terms of the incident pressure $\left(P_{\text {inc }}\right)$, a phase term $\left(e^{i k r}\right)$, the range $(r)$ to account for spherical spreading, and the scattering amplitude $(f)$, which is a complex function of the size, shape, material properties, orientation and acoustic frequency. The scattering characteristics of an object are fully described by the scattering amplitude, the accurate parameterization of which is the focus of scattering physics research.

$$
P_{\text {scat }} \underset{r \rightarrow \infty}{\longrightarrow} P_{i n c} \frac{e^{i k r}}{r} f
$$

While the U.S. Office of Naval Research has funded research into the acoustics of marine mammals, fish and zooplankton, this paper focuses on highlights of fish-related acoustics research. The examples included here are not exhaustive, but are chosen to demonstrate the fundamental physical principles which have shaped the program. The paper is organized as follows: Section II describes laboratory measurement and analysis, Section III illustrates modeling of acoustic scattering by individual organisms, Section IV outlines in situ measurements using broadband signals, and the last section provides a summary. 

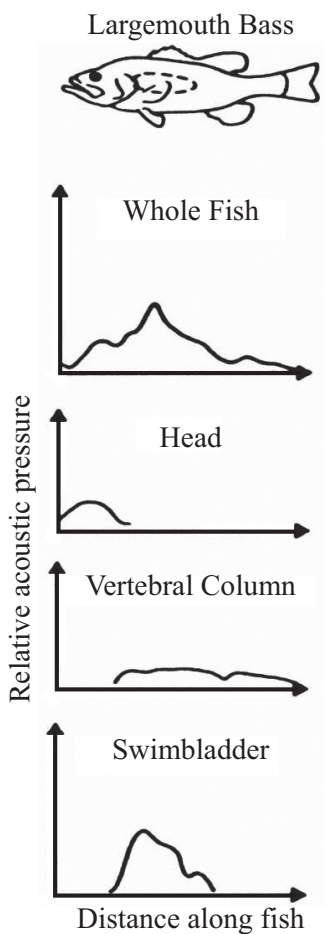

Fig. 1. Relative acoustic pressure as recorded along the length of a large-mouth bass showing relative scattering contributions by the whole fish, head, vertebral column, and swimbladder. This qualitative illustration of the dependence of fish target strength on changes in morphology was generated through the use, in a laboratory, of a focused array transducer system that scanned the length of the fish at $220 \mathrm{kHz}$ in the near field. Adapted from Nash et al. [12].

\section{LABORATORY MEASUREMENT AND ANALYSIS}

Laboratory investigations have included biological and acoustical measurements using narrowband and broadband signals, and advanced signal processing and analysis.

\section{Biological Measurement}

Almost all fish have gas-filled swimbladders, which have been estimated to contribute approximately $90-95 \%$ of the scattering by single fish at broadside incidence [7]. Over the years, quite a bit of effort has been made to characterize the morphological features which drive the dominant scattering mechanisms of marine organisms, from traditional dissection, traditional x-rays [5], CAT (computerized axial tomography) scans, and phase-contrast x-rays [13]. These imaging techniques have been used to create digital representations of fish swimbladders and bodies to provide geometrical inputs to acoustic scattering models.

\section{Acoustical Measurement}

Laboratory measurements of acoustic scattering by fish have been made to ascertain the dominant scattering mechanisms. Laboratory measurements have facilitated the char-
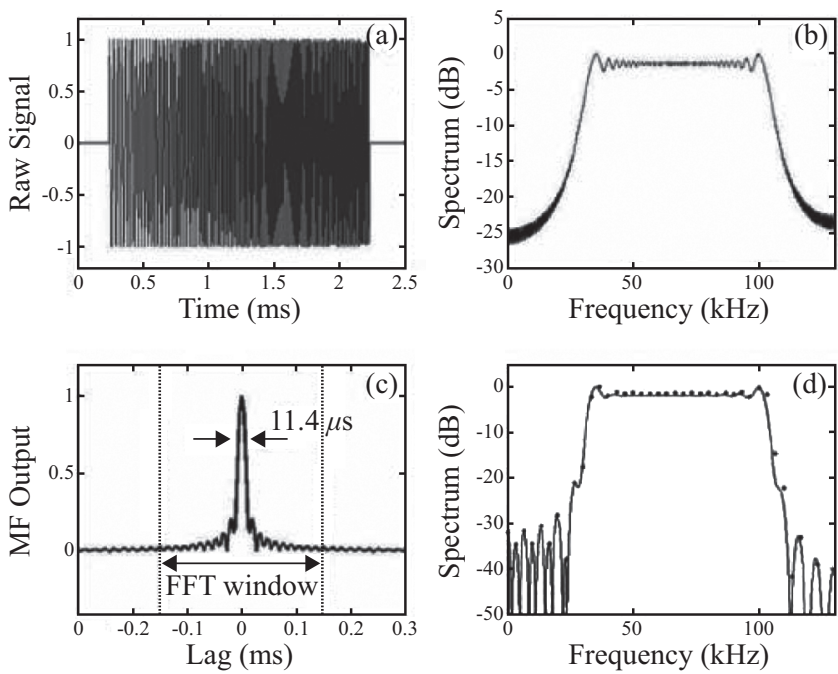

Fig. 2. Compressed pulse processing: Time-series of a $2 \mathrm{~ms}$ duration frequency-modulated (FM) chirp signal (a) having a band of 40-100 kHz as seen in (b). The envelope of the auto-correlation (c) of the time-series signal (a) possesses greater SNR and range/time resolution, and is inversely proportional to the bandwidth (BW). Spectrum (d) of the gated compressed pulse signal, having a spectrum comparable to (b). From Stanton and Chu [15].

acterization and quantification of the acoustic scattering, in terms of angular dependence at different frequencies in the lateral and dorsal/ventral planes [8]. Nash et al. [12] demonstrated that the sum of the scattering by the parts does not equal the scattering by the whole, due to complex constructive and destructive interference occurring within the fish (Fig. 1). While these interference patterns are extremely complex, it is this very complexity that contains the biological information of interest.

\section{Broadband Signals and Pulse Compression Processing}

Since the interference patterns caused by two or more dominant scatterers in the organism vary with frequency, single frequency measurements are not sufficient to accurately characterize the scattering physics. Besides lack of spectral resolution, traditional target strength measurements at single frequencies ignore coherent (phase) information buried in the scattered signal. The use of broadband signals provides greater spectral resolution and allows time-domain processing with matched filters ("compressed pulse output"), which provides higher signal-to-noise ratio (SNR) and greater spatial resolution.

Fig. 2 demonstrates this point. Panel (a) depicts a frequency-modulated (FM) chirp signal having a band of 40-100 $\mathrm{kHz}$ as seen in Panel (b). The envelope of the auto-correlation (Panel (c)) of the time-series signal in Panel (a) is an ideal matched filter or compressed pulse output, possesses greater SNR and range/time resolution, and is inversely proportional to the bandwidth (BW). For this particular FM chirp, the range resolution is approximately $2 \mathrm{~cm}$, which represents a spatial resolution 150 times greater than the original signal [15]. 

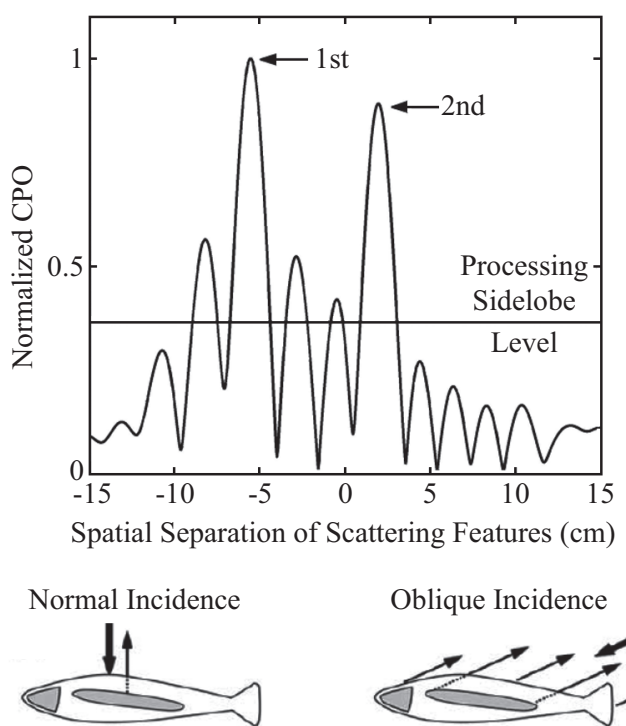

Oblique Incidence

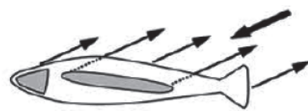

Fig. 3. Envelope of normalized compressed pulse output (CPO) for a fish at approximately $30^{\circ}$ from tail-on orientation in the lateral plane (single-ping realization). The time delay on the horizontal axis has been converted to distance $(\mathrm{cm})$ to represent the spatial separation between the scattering features of the fish, using the equation: separation $=($ time delay $) * c /(2 \cos (\beta))$, where $\beta$ is the angle of orientation relative to the transducer beam and $c$ is the speed of sound. The processing sidelobe is an artifact of the pulse compression process; therefore, any peak significantly higher than the processing sidelobe level represents a physical arrival from the fish. From Reeder et al. [13].

Fig. 3 shows this methodology applied to alewife fish (Alosa pseudoharengus). By cross-correlating the scattered signal with the transmitted signal, the compressed pulse output (CPO) contains distinct and separate arrivals from different parts of the fish. The horizontal scale is converted to spatial separation of the scattering features, and the $8 \mathrm{~cm}$ difference here correlates well with the distance between the skull and the swimbladder of the fish [13].

Taking this a step further, Fig. 4 is a plot of compressed pulse output vs. time lag for each angle as the fish is rotated in the acoustic beam from tail-on aspect toward broadside incidence in the lateral plane; the amplitude is normalized to the maximum for all pings. The bottom axis has been converted to spatial separation $(\mathrm{cm})$ instead of time lag. At tail-on aspect, there is very weak scattering, indicated by the many small amplitude arrivals in the first 20 degrees. As the fish is moved from tail-on toward broadside orientation, the spatial separation of the different parts of the fish relative to the transducers decreases. The first arrival is from the swimbladder and is relatively consistent in space while increasing in amplitude. The multiple and variable arrivals in the center indicate complex constructive and destructive interference as a function of angle. The latest echo, which was initially in the shadow of the swimbladder at tail-on aspect, becomes more significant as broadside incidence is approached. Once near broadside, there is one main arrival as all the energy is scattered back to

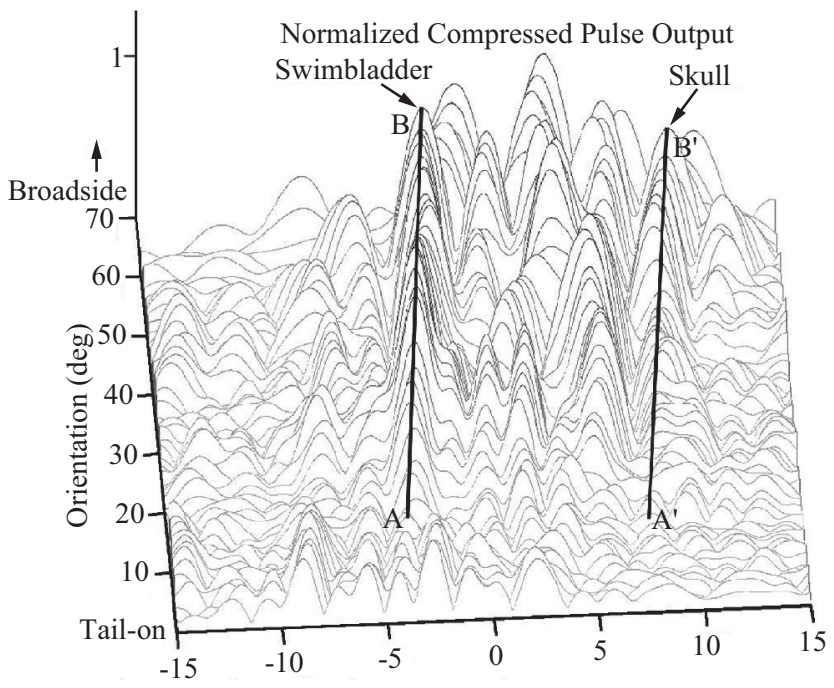

Tail <-- Spatial Separation of Scattering Features $(\mathrm{cm})$--> Head

Fig. 4. Envelope of normalized CPO ("waterfall plot") for a fish as a function of angle of orientation (single ping per orientation) and time delay converted to distance (along fish), depicting the changing $\mathrm{CPO}$ as the fish is rotated from tail-on to broadside orientation in the lateral plane. The conversion of time delay to distance (as in Fig. 3) allows better visualization of the scattering features and their changes as a function of angle of orientation. The conversion changes the appearance of the plot by making features parallel (or nearly so, as indicated by the two lines). Lines $A B$ and A'B' correspond to echoes from the swimbladder and skull, respectively. Also, all values of the CPO are normalized by the same value (global maximum of data) so that directional effects can be better illustrated. From Reeder et al. [13].

the receiver simultaneously. This illustrates not just the angular dependence of the scattering, but the relative contributions of the different scattering mechanisms as a function of angle. It is important to note that Foote [7] explicitly stated that the swimbladder is responsible for $90-95 \%$ of the scattering by fish at broadside incidence-Figs. 3 and 4 demonstrate that other scattering mechanisms may become important at oblique angles. This angular dependence of scattering by fish must be accounted for in some applications (e.g. forwardlooking echosounders).

\section{MODELING THE PHYSICS OF ACOUSTIC SCATTERING}

Physics-based modeling of the acoustic scattering by marine organisms is key to fully understanding the physical scattering mechanisms. To demonstrate this point, the target strength of a canonical gas-filled sphere is computed using a standard, exact spherical model [1] and plotted as a function of frequency in Fig. 5. The spectral shape contains three distinct features: (1) the target strength increases as the fourth power of frequency in the Rayleigh scattering region to the left of the peak; (2) the resonant peak; and (3) the target strength becomes a very weak function of frequency in the geometric 


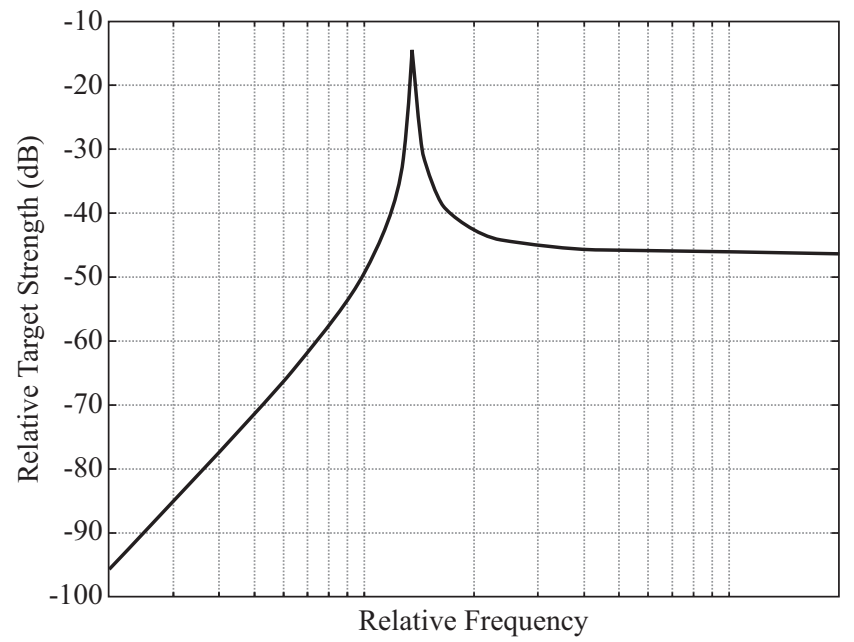

Fig. 5. Relative target strength of a gas-filled sphere as a function of frequency, showing three distinct regions: the Rayleigh scattering region, the resonance peak and the geometric scattering region.

scattering region at frequencies above resonance. Marine organisms are far more complicated than this simple spherical target (as observed by Anderson [1] who developed this model first applied to scattering by marine organisms); however, while extremely complex, modeling the acoustic scattering which depends upon morphology (size and shape), orientation, material properties and frequency of marine organisms complements laboratory measurements and facilitates deeper understanding of the specific scattering mechanisms.

A significant amount of effort has gone into developing scattering models for various classes of marine organisms. A detailed review of the various acoustic scattering models developed for marine organisms is outside the scope of this paper, but is provided in Horne and Clay [10] and Horne and Jech [11]. Acoustic scattering models for fish funded by the Office of Naval Research include the Kirchoff-Ray-Mode model [3-5], the Fourier Matching Method [14] and a hybrid fish scattering model [2]. It is important to keep in mind that, as with all numerical modeling, every model has its specific region of accuracy and validity, as well as inherent limitations. The degree of disparity between predictions and data demonstrates the difficulty in modeling scattering by marine organisms, due to complex morphology, as well as inherent numerical challenges.

\section{IN SITU MEASUREMENT USING BROADBAND SIGNALS}

Traditional fisheries acoustics surveys are carried out at narrowband frequencies in the geometric scattering region (e.g. $18,38,70,120$ and $200 \mathrm{kHz}$ ), and rely upon the relative amplitudes of the backscattered signal at the different narrowband frequencies. Acoustic scattering in the geometric scattering region is not sensitive to frequency (resulting in only small differences in target strength at the different narrowband

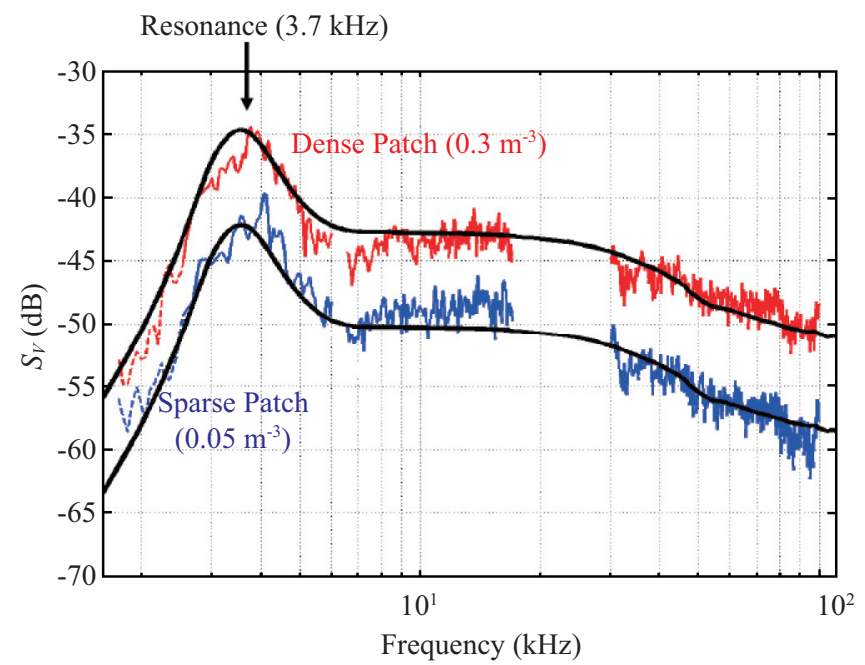

Fig. 6. Resonance phenomenon observed in two separate patches of fish. The red and blue lines represent volume scattering strength (dB) due to dense and sparse patches of fish, respectively. The solid line is a theoretical scattering model. From Stanton et al. [16].

frequencies) but very sensitive to changes in orientation (resulting in large variability in target strength for the same animal on short time scales). However, the resonance frequency of fish swimbladders is sensitive to size (and depth) but insensitive to orientation. Therefore, in the resonance frequency band, knowledge of the animal's depth facilitates inversion for size, no matter the orientation.

The resonance frequency $\left(f_{o}\right)$ of a non-spherical object was parameterized by Weston [18] in terms of shape $(\varepsilon)$, depth $(d)$ and size $(l)$. For many species of adult swimbladdered fish such as Atlantic herring, the resonance frequency occurs in the $1-5 \mathrm{kHz}$ band. In situ investigations of broadband scattering in this frequency band have been heretofore largely technology-limited; however, recent developments in acoustic technology make such investigations possible.

$$
f_{0}=\frac{322 \varepsilon(1+0.1 d)^{5 / 6}}{l}
$$

Fig. 6 shows volume scattering strength $\left(S_{V}\right)$ in $\mathrm{dB}$ as a function of frequency, based upon in situ data collected during a recent experiment using a newly designed towbody equipped with broadband transducers mounted to be downward-looking $[9,16]$. The towbody provides the capability to 'fly' at various depths to better localize the scatterers in the water column. The data demonstrate the expected spectral structure due to backscattering by swimbladdered fish in the shallow water waveguide in the 1-100 $\mathrm{kHz}$ band; they also demonstrate the expected difference in $S_{V}$ due to the presence of adult Atlantic herring in schools of differing numerical density (and not size), as confirmed by concurrent net tows.

The top panels of Fig. 7 show echograms from the same towbody [17]. The echogram on the left shows a near-bottom 

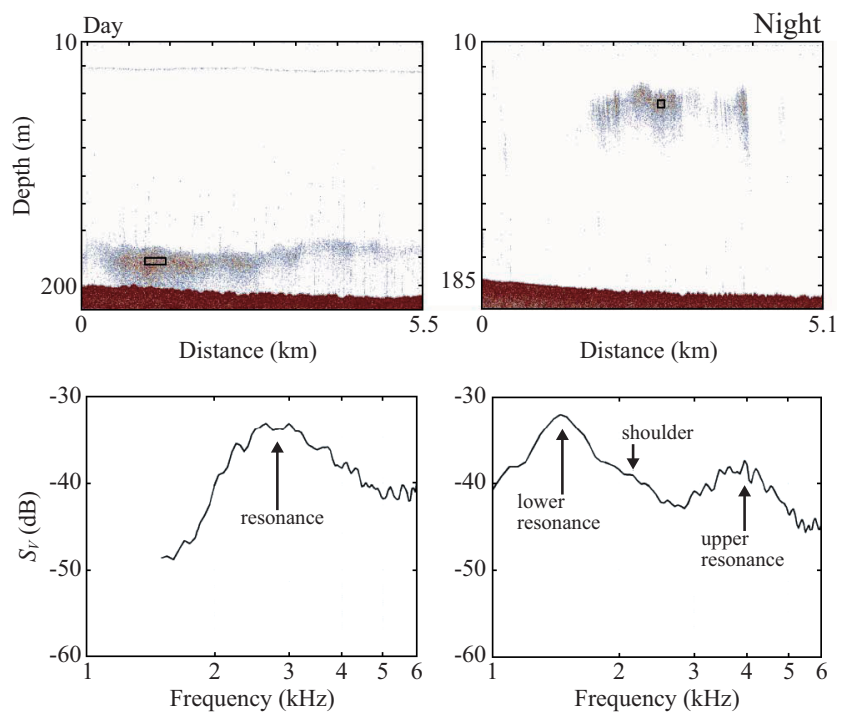

Fig. 7. Echograms of compressed-pulse signals (1-6 kHz) and $S_{V}$ versus frequency for two aggregations: one near the seafloor during the early morning daylight and the other in the upper water column at night. From Stanton et al. [17].

aggregation of Atlantic herring during the day and the echogram on the right shows a near-surface aggregation during the night. These echograms are plots of compressed pulse output from the broadband signals, which possess a range resolution of $20 \mathrm{~cm}$. The corresponding volume scattering strength beneath the left-hand echogram shows a single peak at approximately $3 \mathrm{kHz}$ which corresponds to the resonance frequency of adult herring at depth as confirmed by net tows. The bi-modal distribution in the scattering strength plot on the right corresponds to adult herring (at $\sim 1.5 \mathrm{kHz}$ ) and smaller fish (at $\sim 4 \mathrm{kHz}$ ) in the same aggregation at a more shallow depth, again confirmed by net sampling. This demonstrates the capability to discriminate for size class (and by extension, species) by investigating the resonance frequencies of swimbladdered fish, since the resonance frequency is sensitive to size (and depth) but not angle.

The orientation of the acoustic ensonification of the water column can also be horizontal, which provides a longer range, or larger area, coverage. However, it is important to recognize the challenges presented by longer range in situ measurements - investigators must deal with the multi-scale complexities of acoustic propagation in the shallow water waveguide, as well as the physics of multiple scattering, which can change dramatically with the aggregation's changing position in the waveguide, numerical density distribution and morphology.

Horizontally-oriented acoustic systems also allow investigation and exploitation of the temporal and spectral dependences of the resonance response of various swimbladderbearing fish. Fig. 8 shows the fluctuation in transmission loss (TL) as a function of frequency and time due to aggregations of sardines in the water column between an acoustic source and vertical line array receiver [6]. Several features are

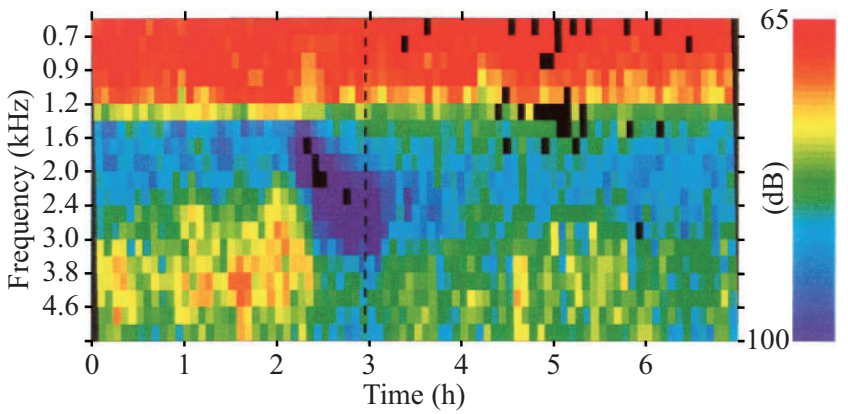

Fig. 8. Transmission loss $(\mathrm{dB})$ as a function of frequency $(\mathrm{kHz})$ and time (hr). Variations in TL are due to changing resonance frequency and school morphology of adult sardines before and after sunrise (indicated by the vertical dashed line). From Diachok [6].

evident in Fig. 8: (1) a broad peak in TL in the $1.5-2.1 \mathrm{kHz}$ band before sunrise, consistent with the swimbladder resonance frequency of dispersed adult sardines at $20 \mathrm{~m}$ water depth; (2) a strong frequency dependence in TL preceding and following sunrise, consistent with the descent of sardines from $20 \mathrm{~m}$ to $65 \mathrm{~m}$ depth (where their resonance frequency is 2.8 $\mathrm{kHz}$ ) followed by school formation (which has a lower resonance frequency than individual sardines); (3) a broad peak in $\mathrm{TL}$ in the $1.8-2.4 \mathrm{kHz}$ band following sunrise, consistent with schooling adult sardines at $65 \mathrm{~m}$ water depth.

\section{CONCLUSION}

Acoustic surveys of marine organisms provide non-invasive, non-destructive, rapid, high-resolution, large area survey capability compared to traditional net tows. However, the ambiguous acoustic data provided by acoustic surveys must be interpreted for meaningful biological parameters. The strategy is to exploit the amplitude, angle and frequency dependence of the scattered signal to infer the size, shape and material properties of individual scatterers. This paper has provided representative highlights from fish acoustics research funded by the U.S. Office of Naval Research which demonstrate the fundamental physical principles which have shaped the program. The program includes laboratory measurement and advanced analysis, modeling of acoustic scattering and in situ measurements using broadband signals and advanced analytical algorithms. Further analysis and modeling will continue to push the science forward to provide robust methods to extract meaningful biological information from acoustic backscattered signals.

\section{REFERENCES}

1. Anderson, V. C., "Sound scattering from a fluid sphere," Journal of the Acoustical Society of America, Vol. 22, pp. 426-431 (1950).

2. Chu, D., Stanton, T. K., Jech, J. M., and Reeder, D. B., "Modeling of the backscattering by swimbladder-bearing fish," Journal of the Acoustical Society of America, Vol. 120, pp. 3105-3105 (2006).

3. Clay, C. S., "Low-resolution acoustic scattering models: Fluid-filled 
cylinders and fish with swimbladders," Journal of the Acoustical Society of America, Vol. 89, pp. 2168-2179 (1991).

4. Clay, C. S., "Composite ray-mode approximations for backscattered sound from gas-filled cylinders and swimbladders," Journal of the Acoustical Society of America, Vol. 92, pp. 2173-2180 (1992).

5. Clay, C. S. and Horne, J. K., "Acoustic models of fish: The Atlantic cod (Gadus morhua)," Journal of the Acoustical Society of America, Vol. 96, pp. 1661-1668 (1994).

6. Diachok, O., "Effects of absorptivity due to fish on transmission loss in shallow water," Journal of the Acoustical Society of America, Vol. 105, No. 4, pp. 2107-2128 (1999).

7. Foote, K. G., "Importance of the swimbladder in acoustic scattering by fish: A comparison of gadoid and mackerel target strengths," Journal of the Acoustical Society of America, Vol. 67, pp. 2084-2089 (1980).

8. Foote, K. G., "Rather-high-frequency sound scattering by swimbladdered fish," Journal of the Acoustical Society of America, Vol. 78, pp. 688-700 (1985).

9. Gauss, R. C., Fialkowski, J. M., Kunz, E. L., Menis, R., Stanton, T. K., Sellers, C. J., and Jech, J. M., "Clutter variability due to fish aggregations: Mid-frequency measurements in the Gulf of Maine," In: Papadakis J. S. and Bjørnø, L. (Eds), Proceedings of the 3rd International Conference on Underwater Acoustic Measurements: Technologies \& Results, F.O.R.T.H., Crete (2009).

10. Horne, J. K. and Clay, C. S., "Sonar systems and aquatic organisms: Matching equipment and model parameters," Canadian Journal of Fisheries and Aquatic Sciences, Vol. 55, pp. 1296-1306 (1988).

11. Horne, J. K. and Jech, J. M., "Models, measures, and visualizations of fish backscatter," In: Medwin H. (written and edited), Sound in the Sea, University Press, Cambridge, pp. 374-397 (2005).

12. Nash, D. M., Sun, Y., and Clay, C. S., "High resolution acoustic structure of fish," ICES Journal of Marine Science, Vol. 44, pp. 23-31 (1987).

13. Reeder, D. B., Jech, J. M., and Stanton, T. K., "Broadband acoustic backscatter and high-resolution morphology of fish: Measurement and modeling," Journal of the Acoustical Society of America, Vol. 116, No. 2, pp. 747-761 (2004).

14. Reeder, D. B. and Stanton, T. K., "Acoustic scattering by axisymmetric finite-length bodies: An extension of a two-dimensional conformal mapping method," Journal of the Acoustical Society of America, Vol. 116, No. 2, pp. 729-746 (2004).

15. Stanton, T. K. and Chu, D., "Calibration of broadband active systems using a single standard spherical target," Journal of the Acoustical Society of America, Vol. 124, pp. 128-136 (2008).

16. Stanton, T. K., Chu, D., Jech, J. M., and Irish, J. D., "New broadband methods for resonance classification and high-resolution imagery of fish with swimbladders using a modified commercial broadband echosounder," ICES Journal of Marine Science, Vol. 67, No. 2, pp. 365-378 (2010).

17. Stanton, T. K., Sellers, C. J., and Jech, J. M., "Resonance classification of mixed assemblages of fish with swimbladders using a broadband acoustic echosounder at 1-6 kHz," Canadian Journal of Fisheries and Aquatic Sciences, under review (2010).

18. Weston, D., "Sound propagation in the presence of bladder fish," In: Albers, V. (Ed.), Underwater Acoustics, Vol. 2, Plenum, New York, pp. 55-58 (1967) 